\title{
Semantic Web Mining in Retail Management System using ANN
}

\author{
Y. Praveen, Suguna
}

\begin{abstract}
Now a day, online shopping is being one of the most common things in the daily lives. To satisfy the customers' requirements knowing the consumer behaviour and interests are more important in the e-commerce environment. Generally, the user behaviour information's are stored on the website server. Data mining approaches are widely preferred for the analysis of user's behaviour. But, the static characterization and sequence of actions are not considered in conventional techniques. In the retail management system, this type of considerations is essential. Based on these considerations, this paper gives detail review about a Semantic web mining based Artificial Neural Network (ANN) for the retail management system. For this review, many sentimental analysis and prediction techniques are observed and compared based on their performance. This survey also focused the dynamic data on the user behaviour. Furthermore, the future direction in big data analytics field is also discussed.
\end{abstract}

Key Words : ANN, Sentimental analysis, big data, Data mining, user behaviour

\section{INTRODUCTION}

critical applications for the future generation of distributed systems are big data analytics. Data mining for such kind of claims presently exceeding Exabyte's and fast increasing in size (kambatla, 2014). Recently, in retail management systems, Big Data is mainly used. The data generation in retails databases are related to variety, veracity, velocity, volume, and value. The process and management of these databases have higher capability compared with the conventional mining methods. Most of the E-commerce companies using different approaches to attract consumers away from the retail outlets by providing some offers like cash back, secure exchange and cash on delivery, etc. So, to survive in this competitive business environment, retailers must identify the problems of their consumers and solve these kinds of issues of the consumers. The different trends in the social media also understand by the retailers on a regular basis. This paper review about the prediction techniques used for the prediction of customer behaviour with some machine learning and deep learning methods. Every transaction made by the customer is stored for Revised Version Manuscript Received on 16 September, 2019.

Y. Praveen, Research scholar, Department of Computer Science Engineering, School of Computing, Vel tech Rangaajan Dr. Sagunthala R\&D Institute of Science and Technology, Avadi, Chennai-62, Tamil Nadu, India

Dr. Suguna, Dept of Computer Science \& Engineering, Vidya Jyothi Institute of Technology, Hyderabad, India observing the purchase pattern of the consumer. Purchase pattern played a vital role in the profit policy for promotion besides placement of the products to fulfil the customer in addition to raise the retailer revenue (Verma et al. 2015). Apriori association algorithm is mostly used to detect the standard items in the databases (Verma and Singh, 2015). However, this method has many limitations such as nature of resource intensive and requirement of multiple scans database. The extraction of unique patterns for buying from big databases also not capable of this method (Malhotra and Rishi 2017). So, the sentiment analysis and prediction methods are used to compare and analyse based on their performance. Sentiment analysis, correspondingly recognized as opinion mining is an important Natural Language Processing (NLP) task that gets much consideration these years, where deep learning based neural network models have attained huge triumph. Sentiment analysis mentions to the procedure of computationally recognizing as well as classifying opinions communicated in a piece of text, in 5 orders to conclude whether the writer's attitude concerning a specific subject or product is positive, negative, or even neutral. In a sentence, not all the words would convey sentimental data. More precisely, only the adjectives, adverbs conjunctions and specific nouns are worthwhile for sentiment analysis. For instances, in view of the subsequent three sentences (i) "I feel very happy about the quality of the product" (ii) "I also felt extreme happy after seeing the price of the product". (iii) "Saying the truth, I have not been pleasant since I was bought this particular product". Both of the sentence (i) and sentence (ii) contain the sentiment keyword "happy" which specifies a positive sentiment. The "happy" seems in two dissimilar positions of different sentences. Also, sentence (iii) encloses two sentiment keywords "not" and "pleasant", which are parted by one more word "been". These two keywords together can accurately show the sentiment extremity of a sentence. In this manner, the essential work for grouping the sentiment keyword of a sentence is to find notion catchphrases precisely. Sentiment ways are anticipated by breaking down the assumption of the substance for watchwords for a specific occasion and applying the expectation calculation to the after effects of examination to foresee the consequences of the following conclusion. In spite of the fact that conclusions can be profoundly precisely anticipated when the machine learning calculation is utilized, in situations where information on wistful ways are not adequate, the exactness of the expectation demonstrate rather turns out to be much lower. Due to this issue, we 
anticipate nostalgic ways through a computation strategy utilizing the weighted qualities as opposed to the machine learning calculation.

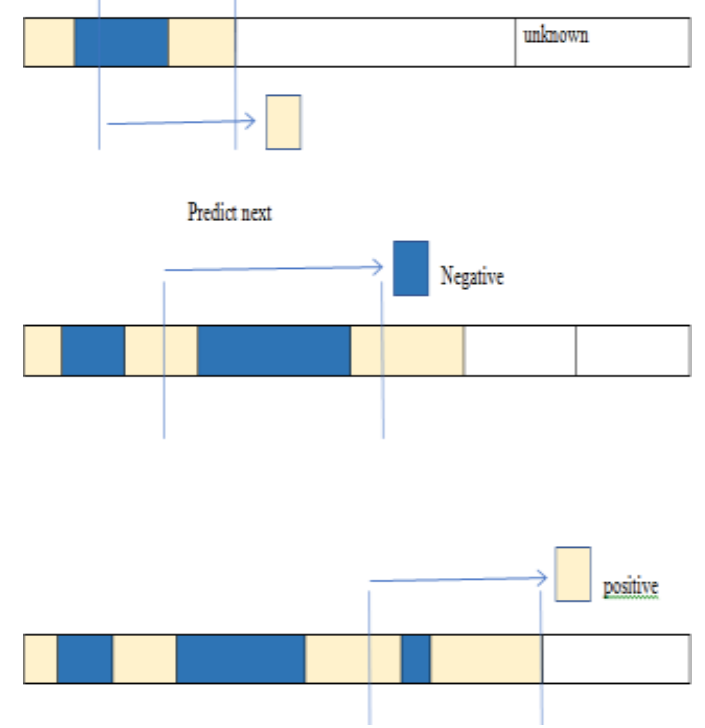

Figure1.The concept of time window

As Figure 1 appears, the idea of time window is utilized for nostalgic way forecast. The measure of the time window can be set in multi day units like 7 days, 14 days, 21 days, and 28 days. We set the window size to 7 days for the analysis. At the point when the time window has been seen as much as the set size, the following estimation is anticipated by the resultant feeling for the pertinent period.

\section{(1/window_size $) \sum$ sentimentpos $\times$ cont_weight

The condition 1 is utilized for sentiment way forecast. Assessments as much as the set time windows are investigated to separate the methods for positive and negative estimations. For this situation, the moving midpoints may be acquired by applying weights, and the resultant qualities utilized for an expectation of following conclusions. Weights (cont_weight) are values somewhere in the range of 0 and 1 for considering the progression of the notions. Be that as it may, there is an impediment of utilizing moving normal to foresee next slant. Since the normal just creates tallies in window smooth, it can't be receptive to dynamic development of qualities. Subsequently, we attempted expectation utilizing LSTM (Long Short-Term Memory) (Greff, Srivastava, Koutnik, Steunebrink, and Schmidhuber, 2016). Figure 6 indicates LSTM for supposition expectation. LSTM is a repetitive neural system design. It is appropriate to anticipate long time arrangement information. Likewise, it has leverage over customary RNNs in light of relative lack of care about hole length (Greff et al., 2016; Hochreiter and Schmidhuber, 1997). So we directed analyses, and utilized LSTM in our expectation as the trial outcome demonstrated that the technique utilizing LSTM was superior to utilizing moving normal.

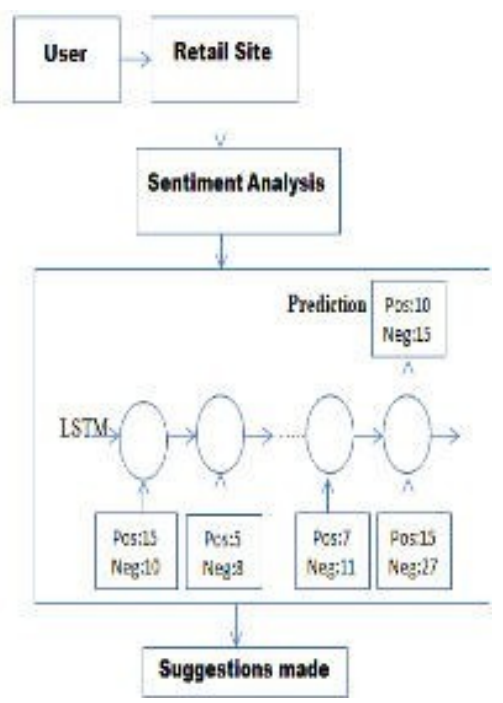

Figure 2 Retail Management System

In figure 2, the user gives their suggestion in the retail site and the sentiment analysis are made and the LSTM artificial neural network prediction algorithm are used to predict the positive and negative value of the feedback and based on that the suggestions can be made. Many papers point out the prediction techniques employed for understanding the customer behavior and re action on a product which are explained in next section. Artificial Neural Network (ANN) is a computational model inspired by the structure and functional aspects of biological neural networks (Coello, 2006). They are useful to learn complex relationships or patterns hidden in large scale semantic data. Researchers have used ANN to enhance ontology alignment (Chortaras et al., 2005; Mao et al., 2010), ontology enrichment (Chifu et al., 2010), concept mining (Honkela et al., 2009), automatic ontology construction(Chen et al., 2008) etc. Supervised ANNs are extensively useful in learning semantic mappings amongst heterogeneous ontologies. Recursive Neural Network (RNN) model (Mandic et al., 2001) was considered to process structured data well appropriate for utilization with ontologies that are in a structured data representation also. RNN was preferred to model automatic ontology alignment (Chortaras et al., 2005). One issue regarding ontology alignment is to discover best configuration that can satisfy ontology constraints, Projective Adaptive Resonance Theory Neural Network (PART) (Cao et al., 2004), was correspondingly used to support automatic ontology construction from web pages (Chen et al., 2008). The PART is accomplished to cluster the web pages which are gathered for the sake of looking for representative terms of every group of web pages. The representative terms are input to a Bayesian. The most representative benefit of CI techniques for the Semantic Web is their ability to tackle difficult issues in an extremely dynamic as well as decentralized setting.

\section{RELATED WORKS}

Yu et al. (2018), demonstrated an online big data-based model for the oil consumption. This model was based on the Google trends. This method also investigated the feasibility of Google 
trends in the online big data prediction for oil usage. For that, this method involved in two steps namely relationship investigation and improvement in the prediction. Granger causality and co integration test were used to predict the Google trends power in the related study statistically. In the prediction improvement step, for oil consumption prediction many classification techniques were introduced. They are logistic regression, decision trees, Support Vector Machine (SVM) and Back Propagation Neural Networks (BPNN).

Johnson et al. (2015), presented an approach to influence the online social networks and recommended a dynamic pricing strategy for the variation in the customer estimation. This approach described a mechanism of dynamic pricing that estimates the customer's interest in the vendor's product. This interest partially imitated the sentiment of the customers towards the products because of the social media. Based on this, the emotion aware pricing method utilized the demand in forecasting including the temporary fluctuation in the customer choice parameters. This parameter was taken from the sentimental dynamics of the social media data. This approach successfully combined with the demand forecasting module of the existing pricing mechanisms. This approach explored the sensitivity of the proposed mechanism performance regarding an error in the sentiment forecasting and simulation. These simulations showed that the forecasting errors underestimated customer sentiment.

Al-Obeidat et al.et al. (2018) presented an Opinion Management Framework with the integration of topic extraction, project management, and sentimental analysis. The comments were placed into the clusters in the topic extraction, and each of the groups was associated with the resolving issues. One or many tasks could be identified from each cluster. The sentiment expression measurement represented the significance of each cluster. This framework recommended the collection of comments about every issue and sentiment was measured from this comments sets. If any subtasks identified within the task, then these also considered. Merchant considered these subtasks and functions and the person who select tasks or subtask was addressed. From these tasks and subtasks, project management features were provided by the vendor. These features were duration and costs, shared resources constraints and earlier start times. This work also considered the task combination and selection of tasks that compensate the performance cost. These optimal selections based on the sentiment improvement in the merchant place value and relation. From this framework, the merchant can immediately respond to the customer's comment online.

Day et al. (2017), applied a deep learning method for the sentiment analysis and focused the consumer review in the mobile phones. For the evaluation and analysis of the consumer review, deep learning method, opinion dictionary and sentiment dictionary were used in the domain of smart phones. In this approach, consumer reviews were collected based on the polarity analysis for smart phone applications. Deep learning method was used to get higher accuracy. Compared with the general machine learning methods the polarity analysis results were best when using the deep learning method.

Due to the economic uniqueness, the automation in the retail trade is very difficult in many business processes. Consider one business process which is a vending machine formation based on fuzzy sequence algorithm. The main problem with the fuzzy logic-based algorithms is its action. A large number of data needed to form the solution in fuzzy logic systems. Generally, the fuzzy logic algorithm requires the period selection analysis and product information. The product information such as purchasing and selling prices, number of elements sold, number of products in the machine. These types of analysis need many hours for the professional marketer, and it takes considerable time, so it is not acceptable.

Semenov et al. (2017), observed these assortments forming problems in the customer demand forecasting. Initially, history of the product was examined then the future behaviour of the product was detected. Finally, the future profit of the machine was predicted using Artificial Intelligence technologies. In this approach, Artificial Neural Network was employed to solve the machine assortment problem.

Wang et al. (2016), compared various predictive methods for house price prediction. ANN performance was compared with the multiple regression analysis (MRA) with the addition of autoregressive integrated moving average approach. The presented model gave high accuracy in the future prediction of prices. Here, housing prices were mentioned in time series formation. This method was observed with housing prices in different places of the world and financial markets. For relationship modelling between prices and quantities, ARIMA model was used in time series. An autoregressive model was used to find the variable value. The relationship between the variable and past period residual was examined by Moving average models.

Ak et al. (2016), compared, two machine learning methods for the estimation of prediction interval (PT) of time series prediction. For prediction quality measurements PI coverage probability andPI width are taken. Initially, Multi-Objective Genetic Algorithm was used to train multilayer perception NN (MLPNN). This method integrated the PI integration in learning and MLPNN was trained for the minimization of width as well as the coverage probability maximization 
in PI estimation. The second method was the combination of Extreme Learning Machines (ELMs) including the Nearest Neighbour Approach. The trained ELMs predicted the estimation points. Then, in training dataset depends on the characteristics of the ELM, PIs were quantified. These two methods were selected for consideration due to the different estimation approaches of PI. For the identification of Pareto front solutions in PIW and PICP, a multi-objective optimization framework was used.

Malhotraet al. (2018), presented an RV-Map reduce big data analytics outline in market basket analysis. By using this framework, the accomplishment of E-commerce websites was easily made based on ranking. This was scalable and robust as well as it was an open-source platform for E-Commerce processing based on big data. Hadoop cluster was described by parallel machines, and big data sets could be easily stored and processed. So, that large number of customers can quickly allow their developments to Hadoop cluster distribution from various locations. This framework recommended that Hadoop and Map-Reduced cloud computing could be preferred for practical deployment in E-commerce ranking system. The primary purpose of the framework was the customer assessment in ranking E-commerce websites and easy searching as well as a perfect ranking of E-Commerce websites.

Chen et al. (2015) proved the need of artificial neural networks in the retail management system comparing with the other methods. However, this approach did not answer the issues related with ANN. Different mixtures of set parameters gave about various resultants such as a structure of input neurons, starting value if height and hidden neurons. Therefore, this proposed method only used the stock closing price as input, and different types of setting parameters were taken for experiments. This paper also enhanced the Back-Propagation Neural Network (BPN) including a new normalized function. BPN minimized the error in the system. MSE and mean absolute percentage error was used for model evaluation. The result provided by this system was better compared with the other systems regarding accuracy.

Lu et al. (2015) observed the variable structures of vegetable price including the weight optimization and BPNN threshold values. Particle Swarm Optimization (PSO) algorithm was utilized to predict the retail price of the vegetables. From the investigational outcomes, it was verified that the PSO-BPNN method normalized the over fitting problem well compared with the traditional back propagation method. This proposed PSO-BP efficiently reduced the training error and improved the precision in prediction.

Thakur et al. (2015) presented a combined approach for gas price prediction with ANN and moving average methods. The input layer, activation function, and hidden layer were employed to achieve output. A neural network trained the neuron numbers which were in the hidden layer. For the measurement of linear and nonlinear series values, neural network and moving average were used. But the neutron in the hidden layer can make an impact in error and reduce the stability as well as over fitting. This model mainly focused on the hidden layer neutron selection, so it resulted in less value in the mean square root value.

Heinrich et al. (2015) showed the dynamic capabilities of big data analytics in the prediction of customer behaviour, adaptive skills, key performance measurement and maintaining the temporary viable advantage of competitors. In this way, the big data value might be deployed for radical and incremental innovations. The additional changes enhanced both the current and existing marketing strategies, and the radical innovations defined a new method for anticipatory shipping strategy. Bekmamedora and Shanks (2014), described the bank social media marketing approach. In this approach, the actions in addition to insights came from big social data were efficiently embedded in the existing business operation and marketing managers' decision-making legacy as well as business analysts. Dutta and Bose (2015) studied the engrossment of the generic business based on the value from the big data analytics. For this, genetic algorithm and BPNN were used to fulfil the deployment and business model enhancement for nine building blocks. This study highlighted the difficulty of the social big data and requirement for mind-set change for marketing heads and employees for any organization. It also presented the application of social big data analytics in the different levels of the production cycle, groundwork identification plan, and strategies in data mining.

Malik et al. (2017) investigated the prediction method based on the impacts of negative and positive reviews about a product. From the review content, the positive and negative emotions were predicted using a deep neural network (DNN). This approach also facilitated the E-commerce retailers and managers in the minimization of the processing cost for getting improved reviews. From the results, it was obtained that the DNN based review prediction technique had vital role compared with the existing prediction techniques.

Wang et al.(2018),suggested a technique for the evaluation of economic sustainability in geographic units based on the convolution neural networks $(\mathrm{CNN})$. This method was introduced to fulfil the gap in the little market estimation issues and provided a sustainable business strategy for retail shops. It was based on the estimation of market demand of the retailers over actual sales data and social media., and it formed a market potential map. By the consideration of spatial proximity, a nuclear density method was implemented. The market potential was estimated by the established model without the knowledge of retailers. For the estimation accuracy verification, the 
presented technique was associated with ANN and least square regression method using cross-validation. The outcomes of proposed technique have greater precision compared with the existing techniques and also it could be applied for the estimation of micro scale market potential.

Krebs et al. (2017), proposed a reaction prediction on the Facebook post by using neural networks. For that, a data set was used to find the Facebook post reaction, and it was useful for both marketing users and machine learners. Then sentimental analysis and emotion mining of Facebook posts was performed by the prediction of user reactions. Initially, emotion mining techniques and emotional analysis were utilized for the sentimental analysis of Facebook comments and posts. Then, NNs including pre trained word embedding's were utilized to approximate the postre action accurately.

Wehrmann et al, (2018) proposed an innovative approach for sorting the sentiment as well as the language of tweets. The proposed architecture included a Convolution Neural Network (ConvNet) by means of two different outputs, each of which considered to reduce the classification error of either sentiment assignment or else language identification. Outcomes presented that the suggested method outperforms both singletask in addition to multi-task advanced approaches for sorting multilingual tweets.

Jianqiang et al, (2018) introduced a word embedding's technique acquired by unsupervised learning based on large twitter corpora with latent contextual semantic relationships and co-occurrence statistical characteristics between words in tweets. These word embedding's are integrated by means of $\mathrm{n}$-grams features in addition to word sentiment polarity score features to form a sentiment feature set of tweets. The feature set is included into a deep CNN for training and forecasting sentiment classification labels. Experimentally compared the performance of the proposed model through the baseline model that is a word n-grams model on five Twitter data sets, the outcomes specified that the proposed model achieved improved on the accuracy and F1-measure for twitter sentiment classification.

Poria et al 2017 presented a multimodal data analysis structure. It incorporated the extraction of remarkable highlights, improvement of unimodal classifiers, building highlight and choice level combination structures. The deep CNN-SVM based sentimental analysis part was observed to be the key component for beating the best in conventional model precision. MKL has assumed a critical part in the fusion experiment. The proposed decision level fusion design was likewise an essential contribution of this research. On account of the decision level fusion experiment, the coupling semantic patterns to decide the heaviness of literary methodology had improved the execution of the multimodal sentimental analysis system significantly. Strangely, a lower precision was gotten for the task of emotion recognition, which may show that extraction of emotions from video might be more troublesome than deducing polarity. While content was the most vital factor for deciding extremity, the visual methodology demonstrates the best execution for feeling examination. The most intriguing part of this paper is that a typical multimodal data analysis structure was well suitable for extraction of emotion and sentiment from various datasets.

The below table shows the summary of different methods involved in big data analytics of retail management system. 
Table 1: Comparison of Different Big Data Analytics in Retail Management System

\begin{tabular}{|c|c|c|}
\hline Authors & Method & Inferences \\
\hline $\begin{array}{l}\text { Verma \& Singh } \\
\text { 017) }\end{array}$ & $\begin{array}{l}\text { 1. Intelligent } \\
\text { adoop Distributed } \\
\text { priori Algorithm } \\
\text { 2. Mapreduce } \\
\text { chitecture }\end{array}$ & $\begin{array}{l}\text { 1. Sales } \\
\text { proved by } \\
\text { pderstanding } \\
\text { sstomer buying } \\
\text { atterns } \\
\text { 2. Customer } \\
\text { ofile analysis } \\
\text { hd browsing } \\
\text { tory identified } \\
\text { ew sales } \\
\text { pportunities }\end{array}$ \\
\hline Poria et al (2017) & Deep CNN-SVM & $\begin{array}{l}\text { High } \\
\text { capability of } \\
\text { extracting } \\
\text { emotion and } \\
\text { sentiment } \\
\text { from various } \\
\text { datasets }\end{array}$ \\
\hline Malik et al. (2017) & $\begin{array}{l}\text { Deep neural } \\
\text { etwork }\end{array}$ & $\begin{array}{l}\text { fluence of } \\
\text { gative and } \\
\text { sitive emotions } \\
\text { product reviews }\end{array}$ \\
\hline $\begin{array}{l}\text { Malhotra and Rishi } \\
\text { 018) }\end{array}$ & $\begin{array}{l}\text { Relevancy vector } \\
\text { lgorithm }\end{array}$ & $\begin{array}{l}\text { ssessed to the } \\
\text { stomer for easy } \\
\text { arching and } \\
\text { ovided a rank } \\
\text { r E-commerce } \\
\text { ebsites based on } \\
\text { eir opinion }\end{array}$ \\
\hline Krebs et al. (2017) & $\begin{array}{l}\text { Deep neural } \\
\text { etworks }\end{array}$ & $\begin{array}{l}\text { atasets were } \\
\text { ed to predict the } \\
\text { stomer reaction } \\
\text { om Facebook } \\
\text { st and } \\
\text { mments. }\end{array}$ \\
\hline Day et al. (2017) & $\begin{array}{l}1 . \\
\text { Recurrent neural } \\
\text { etwork } \\
\text { 2. Long } \\
\text { hort-term memory }\end{array}$ & $\begin{array}{l}\mathrm{NN} \text { was used to } \\
\mathrm{rt} \text { the relevant } \\
\text { larity analysis } \\
\text { d achieved } \\
\text { tter } \\
\text { rformance with } \\
\text { her methods }\end{array}$ \\
\hline $\begin{array}{l}\text { Al-Obeidat et al. } \\
018)\end{array}$ & $\begin{array}{l}\text { Opinion } \\
\text { anagement } \\
\text { ramework (OMF) }\end{array}$ & $\begin{array}{l}\text { OMF was used } \\
\mathrm{r} \text { the integration } \\
\text { topic } \\
\text { traction, project } \\
\text { anagement, and } \\
\text { ntimental } \\
\text { alysis. }\end{array}$ \\
\hline
\end{tabular}

\begin{tabular}{|c|c|c|}
\hline & & $\begin{array}{l}\text { OMF collect the } \\
\text { mments in each } \\
\text { pue, and from } \\
\text { e comments, } \\
\text { ts sentiment } \\
\text { ere measured. }\end{array}$ \\
\hline Ak et al. (2016) & $\begin{array}{l}\text { 1. Multilayer } \\
\text { erceptron } \\
\text { 2.Multi-objective } \\
\text { enetic Algorithms }\end{array}$ & $\begin{array}{l}\text { Multilayer } \\
\text { rceptron } \\
\text { oduces less } \\
\text { ror value than } \\
\text { OGAs } \\
\text { Multilayer } \\
\text { rceptron } \\
\text { vethe less error } \\
\text { lueand } \\
\text { fferentvaluation } \\
\text { iteria for the } \\
\text { st result. }\end{array}$ \\
\hline Wang et al. (2015) & $\begin{array}{l}\text { 1.Artificial Neural } \\
\text { etwork (ANN) } \\
\text { 2.Autoregressive } \\
\text { tegrated moving } \\
\text { average (ARIMA) } \\
\text { odel. }\end{array}$ & $\begin{array}{l}\text { pwer MSE of the } \\
\text { NN } \\
\text { odels presented } \\
\text { e advantage of } \\
\text { NN over } \\
\text { herpredictive } \\
\text { ols. }\end{array}$ \\
\hline Lu et al. (2015) & $\begin{array}{l}\text { 1. PSO-Back } \\
\text { ropagation } \\
\text { ediction } \\
\text { model } \\
\text { 2.Particle Swarm } \\
\text { Optimization } \\
\text { SO)algorithm. }\end{array}$ & $\begin{array}{l}\text { PSOBP } \\
\text { ethod overcome } \\
\text { e overfitting } \\
\text { oblem } \\
\text { d the problem } \\
\text { local minima } \\
\text { Efficiently conde } \\
\text { ining error } \\
\text { improved the } \\
\text { recasting } \\
\text { ecision. } \\
\text { BPNN has } \\
\text { curate square } \\
\text { ot } \\
\text { ean value }\end{array}$ \\
\hline Chen et al. (2015) & $\begin{array}{l}\text { Backpropagation } \\
\text { ural network } \\
\text { PN) }\end{array}$ & $\begin{array}{l}\text { Provided design } \\
\text { tting } \\
\text { rameters in } \\
\text { PN. 2. Better } \\
\text { sults in } \\
\text { e accuracy of } \\
\text { ediction } \\
\text { mpared other } \\
\text { stems }\end{array}$ \\
\hline
\end{tabular}




\begin{tabular}{|c|c|c|}
\hline $\begin{array}{l}\text { Thakur et al. } \\
015)\end{array}$ & $\begin{array}{l}\text { 1.Backpropagation } \\
\text { eural Network } 2 . \\
\text { ultilayer } \\
\text { evenberg-Marquard } \\
\text { algorithm. }\end{array}$ & $\begin{array}{l}\text { eural network } \\
\text { pwed flexibility } \\
\text { ween the inputs } \\
\text { hd outputs. }\end{array}$ \\
\hline
\end{tabular}

From the literature and table.1, it is understood that some limitations. Initially, it needs the selection of the most suitable Google trends, and therefore, a complete study of all Google trends associated to the oil market is a significant concern. Next, some currently emerging predicting tools, particularly the decomposition-andensemble methods, might also be presented to improve the accuracy of prediction. Third,

compared with the other prediction methods semantic web based Back propagation neural network has better performance for the big data analytics in the retail management system. The proposed S-ANN technique provides less means square value compared with the other predictive tool for big data analytics.

\section{EXPERIMENT ON SENTIMENT ANALYSIS:}

The suggested sentiment analysis is based on sentiment models. We consider a sentiment analysis model which demonstrates an accuracy of approximately $84 \%$ as with the a fore mentioned investigational outcomes. Although training with more information is needed to enhance the accuracy, enhanced sentence analysis outcomes can be achieved by allowing for the features of social media contents like social relations. We have investigated along side traditional machine learning methods to authenticate the suggested model. We preferred Naïve-Bayes, SVM, and Random Forest for the traditional machine learning models. We preferred the similar datasets as the suggested model, and trained with the modules of Scikit-learn.

\begin{tabular}{|r|r|r|r|r|}
\hline M Model & $\begin{array}{r}\text { Naïve-B } \\
\text { ayes }\end{array}$ & SVM & $\begin{array}{r}\text { Random } \\
\text { Forest }\end{array}$ & $\begin{array}{r}\text { Proposed } \\
\text { Model }\end{array}$ \\
\hline Precision & 0.76 & 0.77 & 0.76 & 0.839 \\
\hline Recall & 0.76 & 0.77 & 0.76 & 0.845 \\
\hline F-1Score & 0.75 & 0.77 & 0.76 & 0.841 \\
\hline
\end{tabular}

\section{CONCLUSION}

This paper presented a complete review of different data mining techniques available in the retail management system. Various methods for predicting the user behaviour was considered for analysis. From the investigation, it was identified that ANN based semantic web mining method has better accuracy and less mean square value compared with the other conventional predictive tool methods. The existing technique generates numerous iterative overhead results in the analysis. The pattern extraction efficiency is also very less in conventional techniques. Most of the existing mining techniques do not consider the feedback from the user in retail management systems. In the literature, an online big data-driven oil consumption predicting model was described which utilizes Google trends, which marvellously reveal different related factors built on a myriad of search results. This model includes two key steps, relationship analysis and prediction enhancement. But the proposed model still has the relations concerning Google trends and oil consumption will change in extent over time, and could even vanish.

A study of artificial intelligence in the retail management problems is correspondingly deliberated in the collected works. NNs are becoming a significant tool for predicting retail sales. NNs do not work wonders, but if utilized intelligently, they can display startling outcomes. The problems of relearning the NN and the reliance of accuracy and productivity on a hidden layer's number and neurons numbers must be considered.

In one of the paper in the literature, two machine learning approaches namely Multi-Objective Genetic AlgorithmNeural Network (MOGA-NN) and Extreme Learning Machines (ELM) were integrated and associated with the nearest neighbour's method for predicting Prediction Intervals (PIs). This could be mainly advantageous for applications, in which the existing data set is too short to cover all probable patterns or in which the environmental or working conditions vary. Also, MOGA-NN method does not deliberate the systematic study on the influence of the various numbers of hidden neurons on the estimated PIs (e.g., ensemble of NNs). Sustainability study and market demand estimation in the retail industry through a CNN was proposed in the collected works review. Numerous drawbacks also be existent in this study. In the actual situation, the service areas of every retail shops were different. The service area must be well-known and considered to enhance the estimation accuracy. One more important drawback in this study was that the information on social media data was ineffectively used. The spatial locations of social media data were only considered and the semantic information, time, and personal information were overlooked, which were existing and valuable information. To enhance the study outcomes, much data must be considered and the spatial-temporal relationship concerning social media data and region market potential must be determined. Various factors like road connectivity, weather, and purchasing ability, must be added to each geographic cell to achieve precise and exact outcomes.

An intelligent method to Big Data analytics for sustainable retail environment with Apriori-MapReduce framework was discussed in the literature. It is an intelligent HDFS i.e. Hadoop Distributed File System and MapReduce architecture based scalable, parallel next generation Apriori algorithm i.e. MR Apriori algorithm. This study can be prolonged by including support to subsequent generation of big data systems possessing features such as speedy growth in necessary network bandwidth when 
associated with secondary memory storage necessities, drop in iterative analytics overhead by emerging memory computation models so as to keep intermediate outcomes in memory and therefore to overcome different drawback slinked with conventional HDFS like absence of real-time response as needed by framework based on multiple analytic engines. The accuracy of IRM tool in computation of hit count statistic and so on.

The comparison table verified the efficacy of the proposed method. The future research will be focused on the other deep learning methods for sentimental analysis and customer behaviour prediction in the online environment

\section{REFERENCE:}

1. Ak, R., Fink, O., \& Zio, E. (2016). Two machine learning approaches for short-term wind speed time-series prediction. IEEE transactions on neural networks and learning systems, 27(8), 1734-1747.

2. Al-Obeidat, F., Spencer, B., \& Kafeza, E. (2018). The Opinion Management Framework: Identifying and addressing customer concerns extracted from online product reviews. Electronic Commerce Research and Applications, 27, 52-64.

3. Bekmamedova, N., \& Shanks, G. (2014). Social media analytics and business value: a theoretical framework and case study. In System Sciences (HICSS), 2014 47th Hawaii International Conference on (pp. 3728-3737). IEEE.

4. Cao, Y., \& Wu, J. (2004). Dynamics of projective adaptive resonance theory model: the foundation of PART algorithm. IEEE Transactions on Neural Networks, 15(2), 245-260.

5. Chen, C. C., Kuo, C., Kuo, S. Y., \& Chou, Y. H. (2015). Dynamic normalization BPN for stock price forecasting. In Systems, Man, and Cybernetics (SMC), 2015 IEEE International Conference on (pp. 2855-2860). IEEE.

6. Chen, R. C., \& Chuang, C. H. (2008). Automating construction of a domain ontology using a projective adaptive resonance theory neural network and Bayesian network. Expert systems, 25(4), 414-430.

7. Chifu, E. S., \& Letia, I. A. (2010). Self-organizing maps in Web mining and semantic Web. In Self-Organizing Maps. InTech.

8. Chortaras, A., Stamou, G., \& Stafylopatis, A. (2005, September). Learning ontology alignments using recursive neural networks. In International Conference on Artificial Neural Networks (pp. 811-816). Springer, Berlin, Heidelberg.

9. Coello, C. C. (2006). Evolutionary multi-objective optimization: a historical view of the field. IEEE computational intelligence magazine, 1(1), 28-36.

10. Day, M. Y., \& Lin, Y. D. (2017). Deep Learning for Sentiment Analysis on Google Play Consumer Review. In Information Reuse and Integration (IRI), 2017 IEEE International Conference on (pp. 382-388). IEEE.

11. Dutta, D., \& Bose, I. (2015). Managing a big data project: the case of Ramco cements limited. International Journal of Production Economics, 165, 293-306.

12 Greff, K., Srivastava, R. K., Koutník, J., Steunebrink, B. R., \&Schmidhuber, J. (2017). LSTM: A search space odyssey. IEEE transactions on neural networks and learning systems, 28(10), 22222232 .

13. Guille, A., Hacid, H., Favre, C., \& Zighed, D. A. (2013). Information diffusion in online social networks: A survey. $A C M$ Sigmod Record, 42(2), 17-28.

14. Hochreiter, S., \&Schmidhuber, J. (1997). Long short-term memory. Neural computation, 9(8), 1735-1780.

15. Honkela, T., \& Pöllä, M. (2009, June). Concept mining with selforganizing maps for the semantic web. In International Workshop on SelfOrganizing Maps (pp. 98-106). Springer, Berlin, Heidelberg.

16. Jianqiang, Z., Xiaolin, G., \& Xuejun, Z. (2018). Deep Convolution Neural Networks for Twitter Sentiment Analysis. IEEE Access, 6, 23253-23260.

17. Johnson, S. D., \& Ni, K. Y. (2015). A pricing mechanism using social media and web data to infer dynamic consumer valuations. In Big Data (Big Data), 2015 IEEE International Conference on (pp. 2868-2870). IEEE.

18. Kambatla, K., Kollias, G., Kumar, V., \& Grama, A. (2014). Trends in big data analytics. Journal of Parallel and Distributed Computing, 74(7), 2561-2573.

19. Krebs, F., Lubascher, B., Moers, T., Schaap, P., \& Spanakis, G. (2017). Social Emotion Mining Techniques for Facebook Posts Reaction Prediction. arXiv preprint arXiv:1712.03249.

20. Lu, Y. E., Yuping, L., Weihong, L., Qidao, S., Yanqun, L. I. U., \& Xiaoli, Q. (2015). Vegetable Price Prediction Based on PSO-BP Neural Network. In Intelligent Computation Technology and Automation (ICICTA), 2015 8th International Conference on (pp. 1093-1096). IEEE.

\section{AUTHORS PROFILE}

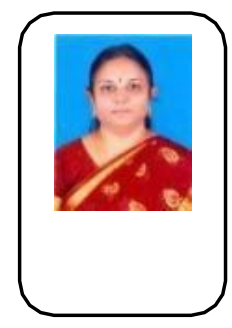

Dr. R. Suguna received B.E. and M.Tech in Computer Science Engineering from Thiagarajar College of Engineering and Indian Institute of Technology Madras during the year 1989 and 2004 respectively. She completed doctorate from Anna University in 2011. She has 22 years of teaching experience and 6 years in industry. She has published $50+$ research papers in reputed journals and conferences. She has delivered invited talks and chaired the sessions in international conferences. Her research interest includes image processing, data mining and machine learning. Currently 10 research scholars are under her supervision. She has organized workshops in machine learning and deep learning in various institutions. She is an active member of IEEE and CSI.

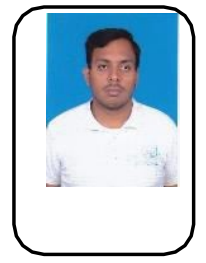

Praveen Kumar Yechuri Research scholar, Department of Computer Science Engineering, School of Computing, Vel tech Rangaajan Dr. Sagunthala R\&D Institute of Science and Technology, Avadi, Chennai-62, Tamilnadu, India\& Asst-Prof, Dept. of CSE, VJIT, HYD. Has 9 Years of Teaching Experience. Has about 8 publications. 